\title{
Drug screening study using glioma stem-like cells
}

\author{
XING FENG, QIN ZHOU, CHONG LIU and MEI-LING TAO \\ College of Medicine, Hunan Normal University, Changsha, Hunan 410006, P.R. China
}

Received May 17, 2012; Accepted August 14, 2012

DOI: $10.3892 / \mathrm{mmr} .2012 .1040$

\begin{abstract}
Glioma is one of the most common malignant tumors affecting the central nervous system. We screened active drugs from 12 classes to provide experimental data for the study of human glioma. Serum-free suspension clone formation was used to induce the formation of glioma stem-like cells (GSLCs) from U251 cells. We screened active drugs from 12 classes and observed their effects on the number of tumor balls. The results revealed that serum-free suspension clone formation successfully induced the formation of GSLCs. Six active drugs [curcumin, chrysin, apigenin, luteolin, casticin and 8-bromo-7-methoxychrysin (BrMChR)] were identified by MTT assay. The drugs that affected the number of tumor balls were curcumin, luteolin, casticin and BrMChR (all $\mathrm{P}<0.05$ ). The GSLCs were obtained by serum-free clone suspension formation and exhibited the features of cancer stem cells.
\end{abstract}

\section{Introduction}

Glioma is one of the most common malignant tumors affecting the central nervous system. In the United States, 22,000 people per year are diagnosed with primary brain tumors (1). Despite a wide range of treatments, including surgery, radiotherapy and chemotherapy, the majority of therapies eventually fail (2). The characteristics of the disease are a short survival period and a high recurrence rate and mortality. Human glioma is defined by the World Health Organization as the most common and malignant type of central nervous system tumor. There is increasing evidence that a variety of cancers, including human glioma, are driven by certain tumor-initiating cells that retain stem cell-like properties. In previous years, researchers have proposed that glioma stem-like cells (GSLCs) not only have similar features to neural stem cells in a number of aspects but also have a certain relationship with embryology (3). Non-Chinese researchers consider GSLCs to be the cause of the occurrence and recurrence of glioma. GSLCs are also the main source of the tolerance of glioma to radiotherapy and

Correspondence to: Mr. Xing Feng, College of Medicine, Hunan Normal University, No. 371 Tongzipo Road, Yuelu District, Changsha, Hunan 410006, P.R. China

E-mail: fengxing01@hotmail.com

Key words: glioma stem-like cells, glioma cells, MTT chemotherapy (4). Therefore, GSLCs have a guiding significance for individual programs of glioma clinical treatment. At present, there are three main methods for separating and identifying GSLCs: serum-free suspension clone formation (5), a CD133 immunomagnetic bead sorting method (6) and flow cytometry of the side population (SP) (7). Temozolomide (TMZ), a DNA-alkylating agent (8), has potent antitumor activity. TMZ is a commonly used neuro-oncology drug. Drug resistance limits the clinical therapeutic effect of this alkylating agent and is one of the primary reasons for the failure of glioma chemotherapy. Therefore, there is an urgent and important requirement for the discovery of new chemotherapy drugs with high selectivity, low toxicity and potent effects for use in the field of brain science.

It has been reported that the growth of tumor cells is inhibited by genistein (9), quercetin (10), resveratrol (11), curcumin (12), chrysin (13), apigenin (14), luteolin (15) and casticin (16). Studies have also shown that the acetoacetate extract of Vitex negundo seed (EVn-50) (17), neolignan (VB-1) (18) and 8-bromo-7-methoxychrysin (BrMChR) (19) are able to inhibit tumor cell growth and promote cell apoptosis. Vitexicarpin, an active component of Vitex trifolia (20) has been reported to induce the apoptosis of breast tumor cells. EFV-3 is an extract of Fructus Viticis. In our study, U251 cells were incubated with serum-free medium. Following the formation of neurosphere-like cells, the active drugs, which are from 12 different classes, were screened by MTT assay. We then observed the effects of these drugs on the number of tumor balls, to provide experimental data for the study of human glioma.

\section{Materials and methods}

Reagents. Dulbecco's minimum essential medium (DMEM) and serum-free Dulbecco's modified Eagle's medium (DMEM)/F12 medium were obtained from Hyclone (Logan, UT, USA). Fetal calf serum (FBS) was purchased from Hangzhou Sijiqing Biological Engineering Materials Co., Ltd. (Hangzhou, China). Trypsin was purchased from Beyotime Biotech (Shanghai, China). Dimethyl sulfoxide (DMSO) was purchased from Genview (USA). 3-(4,5-Dimethylthiazo2-yl)-2,5-diphenyltetrazolium (MTT), genistein, quercetin, resveratrol, curcumin, chrysin, apigenin, luteolin and casticin were purchased from Sigma (St. Louis, MO, USA). EVn-50, VB-1, BrMChR and EFV-3 were gifts from Professor Jianguo Cao (Hunan Normal University College of Medicine). Typan blue, insulin, penicillin and streptomycin were purchased 
from Beijing Dingguo Changsheng Biotech Co., Ltd. (Beijing, China). Cell culture plates and ultra-low attachment plates were purchased from Corning Inc. (Acton, MA, USA). Epidermal growth factor (EGF) and basic fibroblast growth factor (bFGF) were purchased from Protein Specialists (Ness-Ziona, Israel). Genistein, quercetin, resveratrol, curcumin, chrysin, apigenin, luteolin, casticin, EVn-50, VB-1, BrMChR and EFV-3 were dissolved in DMSO. All drug solutions dissolved in DMSO were stored at $-20^{\circ} \mathrm{C}$.

Cell culture and treatment. The U251 cells were obtained from the College of Life Science of Hunan Normal University and were maintained in DMEM supplemented with $10 \% \mathrm{FBS}$, $100 \mathrm{U} / \mathrm{ml}$ penicillin and $100 \mu \mathrm{g} / \mathrm{ml}$ streptomycin at $37^{\circ} \mathrm{C}$ in a humidified atmosphere of $5 \% \mathrm{CO}_{2}$. When the cells were near to $80 \%$ confluence, they were passaged with $0.25 \%$ trypsin. The Ethics Committee of Hunan Normal University approved the study.

Formation of GSLCs. The U251 cells, near $80 \%$ confluence and in good condition, were digested with trypsin and then washed with PBS three times. The U251 cells were seeded in 6-well ultra-low attachment plates at $2 \times 10^{3}$ cells $/ \mathrm{ml}$ in the serum-free stem cell culture medium (DMEM/F12) in the presence of $20 \mathrm{ng} / \mathrm{ml} \mathrm{EGF}$ and bFGF, $4 \mu \mathrm{g} / \mathrm{ml}$ insulin, $100 \mathrm{U} / \mathrm{ml}$ penicillin and $100 \mu \mathrm{g} / \mathrm{ml}$ streptomycin at $37^{\circ} \mathrm{C}$ in a humidified atmosphere of $5 \% \mathrm{CO}_{2}$. Fresh stem cell medium was added every 3 days. Cell growth and cloning ball formation was observed under an inverted microscope after 10 days. The cell culture medium was collected and centrifuged for $5 \mathrm{~min}$ at $800 \mathrm{rpm}$. The cells were digested with trypsin and incubated for 10 days as described above.

Screening active drugs with MTT assay. The U251 GSLCs were seeded in 96-well ultra-low attachment plates at a density of $5 \times 10^{3}$ cells/well and U251 cells were seeded in 96-well plates at the same density. After $24 \mathrm{~h}$, the U251 GSLCs and U251 cells were treated with the 12 drugs. Each drug was tested in three concentrations (10,20 and $40 \mu \mathrm{M})$ and each concentration was used in three parallel wells. After treatment of the cells for $48 \mathrm{~h}, 10 \mu \mathrm{l}$ MTT was added to each well and the cells were cultured for $4 \mathrm{~h}$ at $37^{\circ} \mathrm{C}$. DMSO $(150 \mu \mathrm{l})$ was added to each well. The absorbance at a wavelength of $490 \mathrm{~nm}$ $\left(\mathrm{A}_{490}\right)$ was measured using an enzyme-linked immunosorbent instrument (ELx800, Bio-Tek, Winooski, VT, USA). The experiments were divided into zero setting, control and experimental groups. The relative cell proliferation inhibition rate $(I R)=\left(1\right.$ - average $\mathrm{A}_{490}$ of the experimental group/average $\mathrm{A}_{490}$ of the control group) $\mathrm{x} 100 \%$. The experiment was repeated three times.

Effect on the number of tumor balls. The U251 GSLCs were seeded in 6-well plates. When the cells were near $80 \%$ confluence, the active drugs were applied to treat the cells at a final concentration of $10 \mu \mathrm{mol} / 1$. The cells were digested with trypsin after 48-h treatment and then washed with PBS three times. The cells were seeded in 96-well ultra-low attachment plates at a density of $5 \times 10^{3}$ cells/well. The drug-treated cells were seeded in 96-well ultra-low attachment plates at densities of $5 \times 10^{2}$ and $1 \times 10^{3}$ cells/well. Stem cell medium was added
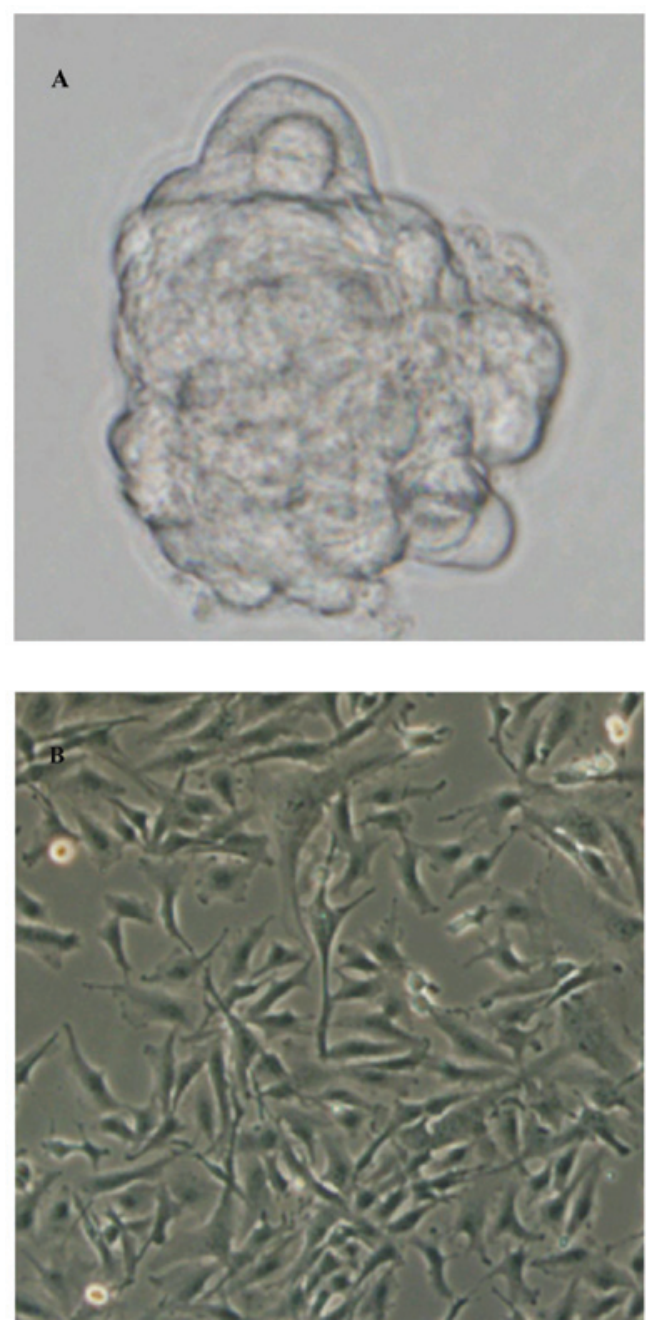

Figure 1. U251 cells and U251 glioma stem-like cells (GSLCs). (A) Serum-free suspension clone formation successfully induces the formation of U251 GSLCs; magnification, $x 40$. (B) U251 cells were adherent to the plastic surface of the cell culture plates grown in DMEM medium containing $10 \%$ FBS; magnification, $\mathrm{x} 10$

every 2 days. The numbers of tumor balls were counted after 7 days.

\section{Results}

Formation of GSLCs. The U251 cells were adherent to the plastic surface of the cell culture plates (Fig. 1B), while the human brain glioma cell lines grew in a globular form in the serum-free stem cell culture medium and successfully formed U251 GSLCs (Fig. 1A). The U251 GSLCs gathered into spherical cell masses of various sizes, suspended in the stem cell medium. The clone balls comprised dozens to hundreds of cells, which had a strong refraction. However, there remained a small number of cells grown in an adherent state. With the prolongation of the time of incubation, we observed the tumor balls gradually becoming larger, with a very clear cell shape and outline, smooth edges, no distinct processes and regular shape. The cells were passaged once every 10 days and the growth process was repeated. The U251 GSLCs had the capacity to proliferate and grow and were able to be passaged continuously. 


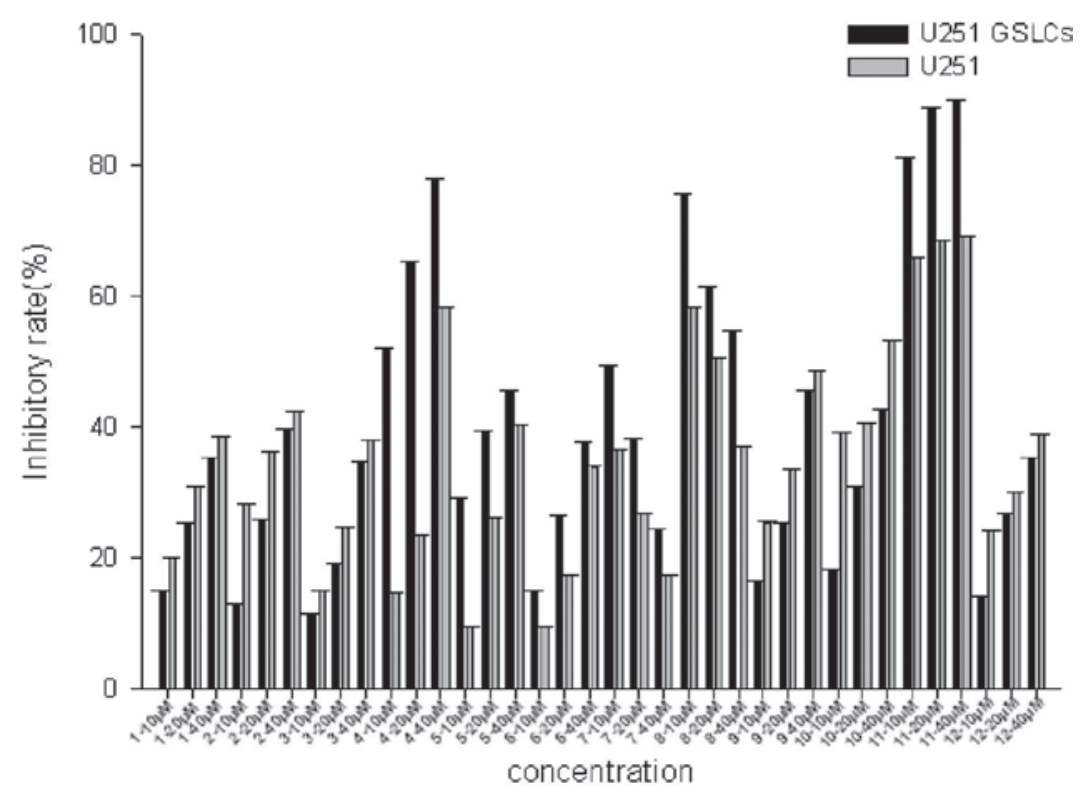

Figure 2. MTT assay screening of active drugs. 1, genistein; 2, quercetin; 3, resveratrol; 4, curcumin; 5, chrysin; 6, apigenin; 7, luteolin; 8, casticin; 9, EVn-50; $10, \mathrm{VB}-1 ; 11, \mathrm{BrMChR} ; 12$, EFV-3. MTT assay showed that the inhibition rate of active drugs from 6 classes to U251 GSLCs is higher than U251 cells, including curcumin, chrysin, apigenin, luteolin, casticin and BrMChR. GSLCs, glioma stem-like cells; MTT, 3-(4,5-dimethylthiazo-2-yl)-2,5-diphenyltetrazolium; EVn-50, acetoacetate extract of Vitex negundo seed; VB-1, neolignan; BrMChR, 8-bromo-7-methoxychrysin; EFV-3, extract of Fructus Viticis.

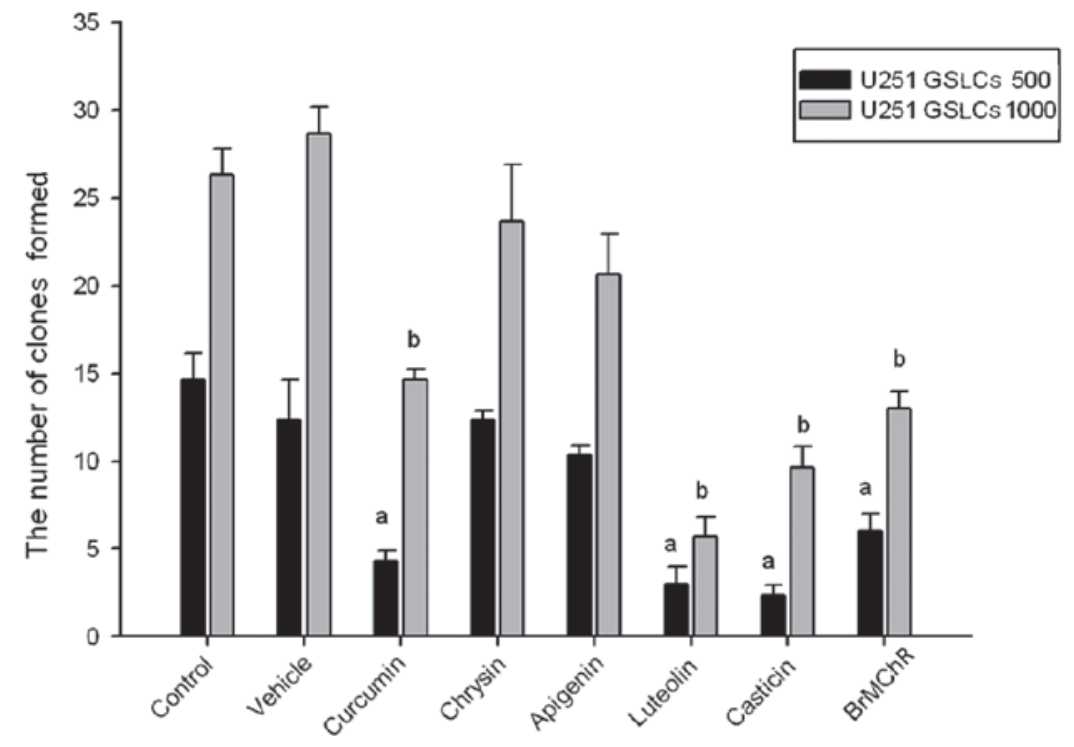

Figure 3. The effect of different drugs on the number of tumor balls. Four drugs affected the number of tumor balls, specifically curcumin $(\mathrm{P}<0.05)$, luteolin $(\mathrm{P}<0.05)$, casticin $(\mathrm{P}<0.05)$ and $\mathrm{BrMChR}(\mathrm{P}<0.05)$, compared with the control group. ${ }^{\mathrm{a}} \mathrm{P}<0.05$ vs. control $\mathrm{U} 251 \mathrm{GSLCs} 500 ;{ }^{\mathrm{b}} \mathrm{P}<0.05$ vs. control U251 GSLCs 1000 . GLSCs, glioma stem-like cells; BrMChR, 8-bromo-7-methoxychrysin. GSLCs 500, 500 U251 GSCLs seeded in 96-well ultra-low attachment plates; GSLCs 1000, 1000 U251 GSCLs seeded in 96-well ultra-low attachment plates.

MTT assay screening of active drugs. The inhibitory rates of drugs from 12 different classes on tumor stem cell-like cells and tumor cells were compared by MTT assay. The results revealed that 6 of the drugs had higher inhibitory rates on the U251 GSLCs than on the U251 cells; these drugs were curcumin, chrysin, apigenin, luteolin, casticin and BrMChR (Fig. 2).

Effect on the number of tumor balls. As shown in Fig. 3, U251 GSCLs treated with 6 active drugs were seeded in 96-well ultra-low attachment plates. The number of tumor balls in the control samples gradually increased over 7 days. The number of tumor balls was decreased by treatment with 4 of the drugs after 7 days, specifically curcumin, luteolin, casticin and BrMChR; all $\mathrm{P}<0.05$ compared with the control group.

\section{Discussion}

Glioma is a common type of adult malignancy with high morbidity and mortality. Although clinical treatments with surgery, chemotherapy, radiotherapy and biological therapy, are presently used, the tumors recur without exception (21-24). 
The disease progresses rapidly following recurrence and seriously threatens the life and health of the patients $(25,26)$. Within the past decades, accumulating evidence from a number of biological systems, including the blood (27), breast (28) and brain $(6,29)$, has indicated that the transformation of cancer stem-like cells may induce the formation of tumors. One novel treatment strategy under investigation is to make cancer stemlike cells differentiate into non-dividing cells. If successful, patients with brain tumors would be able to lead a normal life. The tumor would stop growing due to the terminal differentiation of the cells (30). A number of sorting technologies for stem cell-like cells, including immunomagnetic beads and SP sorting methods, are becoming widely used in the study of cancer stem cell-like cells. However, these sorting methods have a number of limitations.

In our study, serum-free suspension clone formation successfully induced the formation of U251 GSLCs. Purified U251 GSLCs could be suspended in serum-free medium. Active drugs of 6 classes (curcumin, chrysin, apigenin, luteolin, casticin and BrMChR) were identified by the screening of drugs from 12 classes by MTT assay. Four of the drugs were able to affect the number of tumor balls; these were curcumin, luteolin, casticin and BrMChR (all $\mathrm{P}<0.05$ ).

We have preliminarily identified methods for culturing stem cell-like cells and used the cells in the screening of active drugs. The immunofluorescence identification of relevant molecular markers of GSLCs and animal experiments in vivo are subjects of our future studies. Only by performing these studies are we likely to find the most active drug, to contribute further to the investigation of human glioma.

\section{Acknowledgements}

This project was supported by the Provincial Science and Technology Plan of Hunan, China (No. 2010FJ3017), the Research Foundation of Education Bureau of Hunan Province, China (No. 11B081), the Chinese Traditional Medicine Administration of Hunan Province, China (No. 2009102)and the Excellent Talent Program of Hunan Normal University (2011).

\section{References}

1. 2010 CBTRUS Statistical Report: Primary brain and central nervous system tumors diagnosed in the United States in 2004-2006. In: Central Brain Tumor Registry of the United States. http://www.cbtrus.org/reports/reports.html. Accessed March 2010.

2. Gong X, Schwartz PH, Linskey ME and Bota DA: Neural stem/progenitors and glioma stem-like cells have differential sensitivity to chemotherapy. Neurology 76: 1126-1134, 2011.

3. Bearzatto A, Szadkowski M, MaePherson P, et al: Epgenetieregulation of the MGMT and hMSH6 DNA repair genes in cells resistant to methylating Agents. Cancer Res 60 3262-3270, 2000.

4. Lavon I, Fuchs D, Zrihan D, et al: Novel mechanism whereby nuclear factor kappa B mediates DNA damage repair through regulation of $\mathrm{O}(6)$-methylguanine-DNA-methyltrans-ferase. Cancer Res 67: 8952-8959, 2010.

5. Inagaki A, Soeda A, Oka N, et al: Long-term maintenance of brain tumor stem cell properties under at non-adherent and adherent culture conditions. Biochem Biophys Res Commun 361 586-592, 2007.

6. Singh SK, Hawkins C, Clarke ID, et al: Identification of human brain tumor initiating cells. Nature 432: 396-401, 2004.
7. Harris MA, Yang H, Low BE, et al: Cancer stem cells are enriched in the side population cells in a mouse model of glioma. Cancer Res 68: 10051-10059, 2008.

8. Ostermann S, Csajka C, Buclin T, et al: Plasma and cerebrospinal fluid population pharmacokinetics of temozolomide in malignant glioma patients. Clin Cancer Res 10: 3728-3736, 2004.

9. Qi W, Weber CR, Wasland K and Savkovic SD: Genistein inhibits proliferation of colon cancer cells by attenuating a negative effect of epidermal growth factor on tumor suppressor FOXO3 activity. BMC Cancer 11: 219, 2011.

10. Jeong JH, An JY, Kwon YT, Rhee JG and Lee YJ: Effects of low dose quercetin: cancer cell-specific inhibition of cell cycle progression. J Cell Biochem 106: 73-82, 2009.

11. Fukui M, Yamabe N, Kang KS and Zhu BT: Growth-stimulatory effect of resveratrol in human cancer cells. Mol Carcinog 49: 750-759, 2010

12. Ravindran J, Prasad S and Aggarwal BB: Curcumin and cancer cells: how many ways can curry kill tumor cells selectively? AAPS J 11: 495-510, 2009.

13. Brechbuhl HM, Kachadourian R, Min E, Chan D and Day BJ: Chrysin enhances doxorubicin-induced cytotoxicity in human lung epithelial cancer cell lines: the role of glutathione. Toxicol Appl Pharmacol 258: 1-9, 2012.

14. Mak P, Leung YK, Tang WY, Harwood C and Ho SM: Apigenin suppresses cancer cell growth through ERbeta. Neoplasia 8: 896-904, 2006

15. Lin Y, Shi R, Wang X and Shen HM: Luteolin, a flavonoid with potential for cancer prevention and therapy. Curr Cancer Drug Targets 8: 634-646, 2008.

16. Chen D, Cao J, Tian L, Liu F and Sheng X: Induction of apoptosis by casticin in cervical cancer cells through reactive oxygen species-mediated mitochondrial signaling pathways. Oncol Rep 26: 1287-1294, 2011.

17. Xiao JW, Zhuang YZ, Cao JG, et al: Effect of Semen Viticis negundo extract $\mathrm{EVn}-50$ on cell proliferation and apoptosis of human breast cancer cell line MCF-7. Chin J Cancer Prev Treat 16: 175-178, 2009.

18. Zeng FX, Zhou YJ, Tang AQ, et al: Induction of apoptosis in ovarian cancer CoC1 cells by neolignan VB-1. J Hunan Normal Univ (Med Sci) 8: 40-42, 2011

19. Zheng X, Meng WD, Xu YY, et al: Synthesis and anticancer effect of chrysin derivatives. Bioorg Med Chem Lett 13: 881-884, 2003.

20. Song YC, Zhang X, Lei GY and Dang CX: Vitexicarpin affects proliferation and apoptosis in mutated p53 breast cancer cell. Zhonghua Yi Xue Za Zhi 90: 703-707, 2010 (In Chinese).

21. Chang SM, Theodosopoulos P, Lamborn K, et al: Temozolomide in the treatment of recurrent malignant glioma. J Cancer 100: 605-611, 2004.

22. Tatter SB: Recurrent malignant glioma in adults. Curr Treat Options Oncol 3: 509-524, 2002.

23. Wen PY and Kesari S: Malignant gliomas in adults. N Engl J Med 359: 492-507, 2008.

24. Stupp R, Mason WP, van den Bent MJ, et al: Radiotherapy plus concomitant and adjuvant temozolomide for glioblastoma. N Engl J Med 352: 987-996, 2005.

25. Schmidinger M, Linzmayer L, Becherer A, et al: Psychometric and quality-of-life assessment in long-term glioblastoma survivors. J Neurooncol 63: 55-61, 2003.

26. Corn BW, Wang M, Fox S, et al: Health related quality of life and cognitive status in patients with glioblastoma multiforme receiving escalating doses of conformal three dimensional radiation on RTOG 98-03. J Neurooncol 95: 247-257, 2009.

27. Lapidot T, Sirard C, Vormoor J, et al: A cell initiating human acute myeloid leukaemia after transplantation into SCID mice. Nature 367: 645-648, 1994.

28. Al-Hajj M, Wicha MS, Benito-Hernandez A, Morrison SJ and Clarke MF: Prospective identification of tumorigenic breast cancer cells. Proc Natl Acad Sci USA 100: 3983-3988, 2003.

29. Taylor MD, Poppleton H, Fuller C, et al: Radial glia cells are candidate stem cells of ependymoma. Cancer Cell 8: 323-335, 2005.

30. Piccirillo SG, Reynolds BA, Zanetti N, et al: Bone morphogenetic proteins inhibit the tumorigenic potential of human brain tumour-initiating cells. Nature 444: 761-765, 2006. 